This item was submitted to Loughborough's Research Repository by the author.

Items in Figshare are protected by copyright, with all rights reserved, unless otherwise indicated.

\title{
Measurement and modeling of the acoustic field near an underwater vehicle and implications for acoustic source localization
}

PLEASE CITE THE PUBLISHED VERSION

PUBLISHER

(C) Acoustical Society of America

LICENCE

CC BY-NC-ND 4.0

\section{REPOSITORY RECORD}

Lepper, Paul A., and Gerald L. D'Spain. 2019. "Measurement and Modeling of the Acoustic Field Near an Underwater Vehicle and Implications for Acoustic Source Localization". figshare.

https://hdl.handle.net/2134/3828. 
This item was submitted to Loughborough's Institutional Repository (https://dspace.lboro.ac.uk/) by the author and is made available under the following Creative Commons Licence conditions.

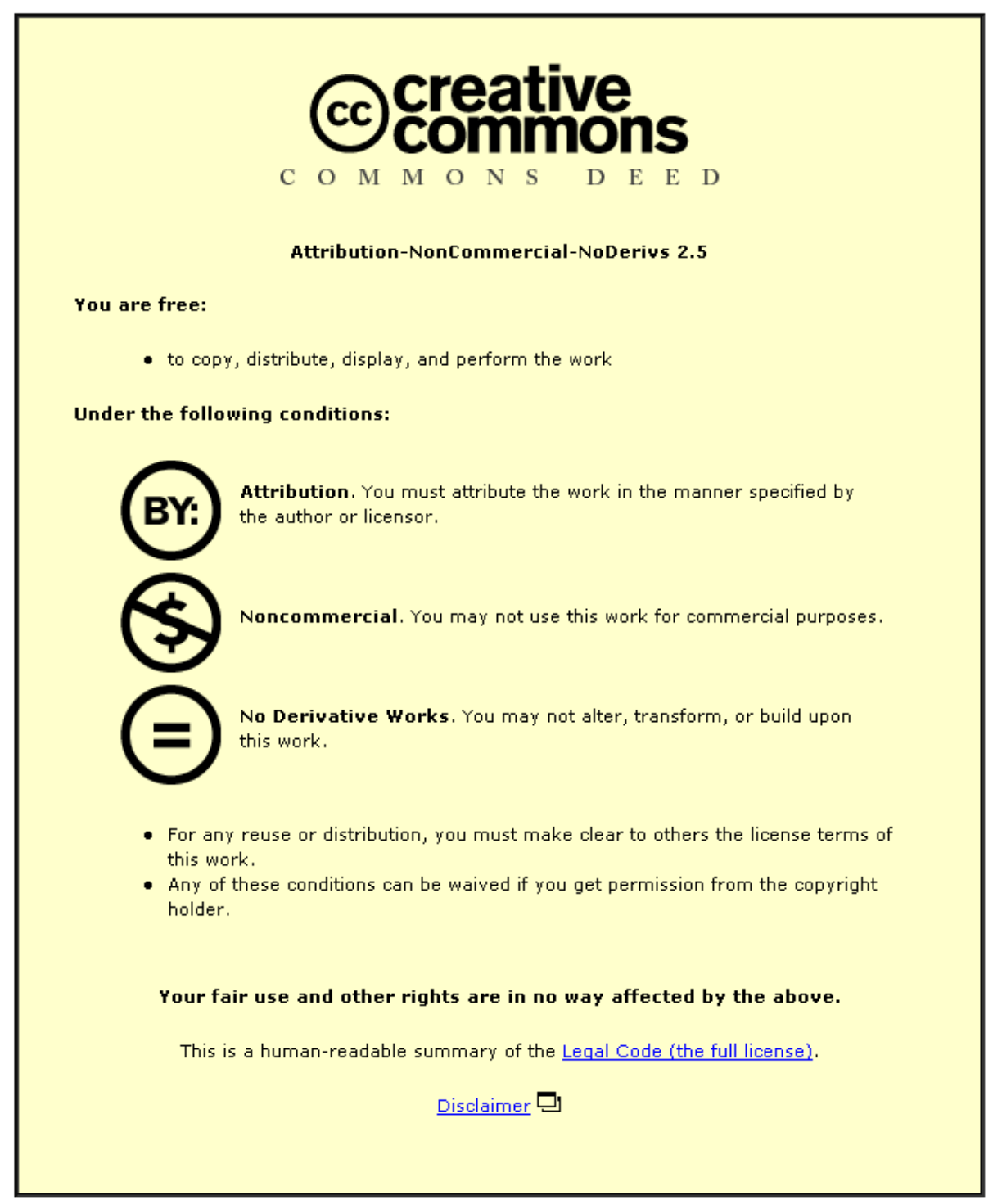

For the full text of this licence, please go to: http://creativecommons.org/licenses/by-nc-nd/2.5/ 


\title{
Measurement and modeling of the acoustic field near an underwater vehicle and implications for acoustic source localization
}

\author{
Paul A. Lepper ${ }^{a)}$ and Gerald L. D'Spain \\ Marine Physical Laboratory, Scripps Institution of Oceanography, La Jolla. California 92093
}

(Received 5 August 2006; revised 17 May 2007; accepted 18 May 2007)

\begin{abstract}
The performance of traditional techniques of passive localization in ocean acoustics such as time-of-arrival (phase differences) and amplitude ratios measured by multiple receivers may be degraded when the receivers are placed on an underwater vehicle due to effects of scattering. However, knowledge of the interference pattern caused by scattering provides a potential enhancement to traditional source localization techniques. Results based on a study using data from a multi-element receiving array mounted on the inner shroud of an autonomous underwater vehicle show that scattering causes the localization ambiguities (side lobes) to decrease in overall level and to move closer to the true source location, thereby improving localization performance, for signals in the frequency band $2-8 \mathrm{kHz}$. These measurements are compared with numerical modeling results from a two-dimensional time domain finite difference scheme for scattering from two fluid-loaded cylindrical shells. Measured and numerically modeled results are presented for multiple source aspect angles and frequencies. Matched field processing techniques quantify the source localization capabilities for both measurements and numerical modeling output. (C) 2007 Acoustical Society of America. [DOI: 10.1121/1.2749410]
\end{abstract}

PACS number(s): 43.60.Kx, 43.60.Fg, 43.60.Jn, 43.30.Wi [EJS]

Pages: 892-905

\section{INTRODUCTION}

Effects such as diffraction and scattering by an object placed in an acoustic field can result in a complex field structure around that object. Diffracted energy from a source may be observed in the geometrical shadow zone behind an object and so cannot be described by simple ray-based modeling. This energy can also interact with the incident signal energy outside the shadow zone, resulting in interference fields that are observed in conventional optics and acoustics (Morse and Ingrad, 1986 and Skelton et al., 1997). The resulting interference patterns often exhibit strong spatial, angular, and spectral dependence for particular source-scatterer-receiver geometries and therefore may provide valuable information regarding the source location.

An investigation of the complex field around two spheres at multiple source aspect angles and signal frequencies has been carried out using both measured data and numerical modeling results. The measurements were made by an eight-element hydrophone array mounted on the Marine Physical Laboratory's Odyssey IIb autonomous underwater vehicle (AUV). The body of this vehicle is free flooding with a majority of the control and instrumentation electronics contained in two 17-in. $(43.2 \mathrm{~cm})$-diameter air-filled glass spheres. The outer shell of the vehicle is a thin walled $(3.5 \mathrm{~mm})$ shroud of high-density polyethylene. For the numerical modeling, the shroud is assumed to be acoustically transparent at the frequencies of interest and the glass spheres are considered to be the major acoustic scatterers.

\footnotetext{
a) Author to whom correspondence should be addressed. Electronic mail: p.a.lepper@lboro.ac.uk
}

Motivation for this study was provided by the results from the field of human hearing. Studies with humans have shown that the spectral patterns resulting from the interference of the direct path arrival with sound scattered from the head, torso, and pinna provide important cues for source localization (Blauert, 1983). In particular, for sound sources that vary in elevation angle in the median sagittal plane of the human body, a notch occurs in the head-related transfer function that varies in frequency (from about 6 to $10 \mathrm{kHz}$ ) in a sensitive way with changes in elevation angle (Butler and Balendiuk, 1997). Therefore, the two instrumentation glass spheres in the AUV used in this study can be viewed as playing the role of two heads.

The aim of this paper is to examine source localization performance in the presence of scattering from the platform making the measurements. In Sec. II, the measurements of the pressure field using an eight-element hydrophone array mounted on the Odyssey IIb are presented. These measurements were made at the Transducer Evaluation Center (TRANSDEC) of the Space and Naval Warfare Systems Center, San Diego, where data for multiple source aspect angles and for frequencies in the range of $70 \mathrm{~Hz}-8 \mathrm{kHz}$ were collected. Section III outlines a numerical modeling effort using CABRILLO (Gerstoft, 2004), a two-dimensional (2D) time domain finite difference scheme, (Luebbers and Beggs, 1992 and Yee, 1966). A comparison between measured data and the numerical modeling results is given in Sec. IV for the midfrequency $(2-8 \mathrm{kHz})$ tone bursts. The disadvantage of the comparison is that the $2 \mathrm{D}$ modeling approach does not account for the three-dimensional (3D) configuration of the AUV or the potential elastic scattering effects of the spheres. However, the results presented do 


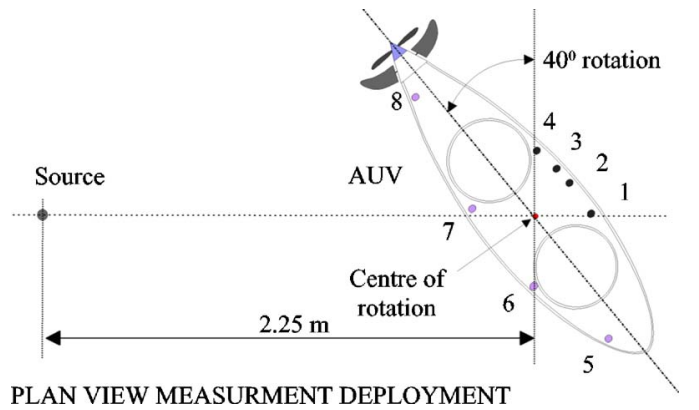

FIG. 1. (Color online) The plan view of the experimental configuration for the measurement effort at the TRANSDEC transducer calibration facility.

illustrate many major physical effects that contribute to the complex acoustic field structure around the AUV. The use of these fields for source localization is then discussed in Sec. V. Results are quantified using matched field processing techniques. Finally, conclusions from this work are given in Sec. VI.

\section{DESCRIPTION OF THE MEASUREMENTS}

\section{A. Experimental setup}

Measurements of the acoustic pressure field surrounding the Marine Physical Laboratory's AUV were made at the TRANSDEC facility, a 22 million 1 fresh water tank, comprising a 53-m-diam inner pool and a canted, ellipse-shaped outer section. The depth of the inner pool varies from $12 \mathrm{~m}$ in the center to less than $1 \mathrm{~m}$ at the edge to minimize acoustic reflections from the edge of the pool. The ellipsed-shaped outer section also acts as an acoustic trap.

Figure 1 shows a schematic of the AUV deployment at TRANSDEC. The vehicle was placed in the center of the tank at the mid-water depth of $6 \mathrm{~m}$. An omni-directional acoustic source was placed at a horizontal range of $2.25 \mathrm{~m}$ from the center of rotation of the AUV body (displaced $4 \mathrm{~cm}$ aft along the main axis of the AUV from the point equidistant between the two glass spheres). The vehicle was suspended from a single rigid shaft linked to a geared stepper motor, allowing precise control of the source / AUV aspect angle in the horizontal plane over a $360^{\circ}$ sector. Figure 2 shows a side view of the AUV. All receivers were placed in approximately the same horizontal plane corresponding to the equatorial plane of symmetry of the two glass spheres and within the outer polyethylene shell of the AUV.

The data acquisition system installed in one of the glass spheres was a PC-104+ based digital recording system capable of collecting up to eight channels of data. Eight High

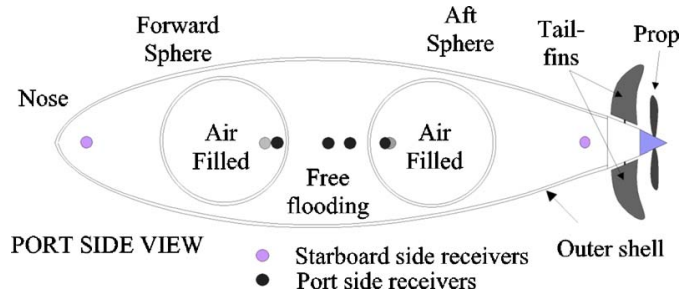

FIG. 2. (Color online) Port-side view of the AUV hydrophone configuration during the measurement effort at TRANSDEC.
Tech, Inc. HTI-94-SSQ hydrophones with built-in $18 \mathrm{~dB}$ preamplifiers were used as the array elements. The analog output from each hydrophone/preamp assembly was bandpass filtered between $20 \mathrm{~Hz}$ and $10 \mathrm{kHz}$ and amplified by $20 \mathrm{~dB}$ for an overall channel sensitivity of $-160 \mathrm{~dB}$ re $1 \mathrm{~V} / \mu \mathrm{Pa}$. The resulting filtered and amplified analog signals were sent to a common analog-to-digital converter, which provided 16 bit data samples from each channel at a $20 \mathrm{ksamples} / \mathrm{s}$ sampling rate. These digital data then were written directly to hard disk. The DAQ electronics were housed in the aft sphere in the AUV along with an independent battery power supply allowing continuous recording for up to $4 \mathrm{~h}$. Further details on the hydrophone array and data acquisition system are provided in Zimmerman et al., 2005.

\section{B. Experimental procedure}

Pulsed continuous wave (cw) combs were transmitted in the low (less than $1 \mathrm{kHz}$ ) and mid $(1-10 \mathrm{kHz})$ frequency ranges using a USRD-J15 moving coil source and a ITC1007 ceramic transducer, respectively. Each of the pulsed comb signals was composed of a linear sum of pulsed $\mathrm{cw}$ tones. For the mid-frequency comb signals, a pulse was $6.4 \mathrm{~ms}$ in duration, corresponding to the time difference between the direct path arrival and the arrival of the first reflections from the surface and tank bottom. The lowfrequency comb was transmitted as both pulsed with $12.8 \mathrm{~ms}$ duration (the longer pulse length was required to achieve the frequency resolution necessary to separate the low-frequency tones) and as a steady-state cw comb.

During each test, the AUV was rotated continuously through a $360^{\circ}$ sector at an angular speed of either $0.2 \mathrm{deg} \mathrm{s}^{-1}$ or $0.4 \mathrm{deg} \mathrm{s}^{-1}$. This rotation rate provided a $0.2^{\circ}$ or $0.4^{\circ}$ angular resolution, respectively. At the higher rotation rate, the variation of $2.56 \times 10^{-3} \mathrm{deg}$ during the measurement period of $6.4 \mathrm{~ms}$ was considered insignificant. Tests were conducted over a $360^{\circ}$ sector for both comb signals. Each test was started with the AUV at broadside $\left(0^{\circ}\right)$ to the source with receivers 5 to 8 (starboard side) closest to the source, as shown in Fig. 1. The AUV was rotated counter-clockwise through positive angles with the propellor of the AUV closest to the source at an angle of $+90^{\circ}$ and the nose closest at $+270^{\circ}$.

\section{Signal analysis}

Figure 3 shows the time-frequency response for the midfrequency comb $(1900,2925,4060,5100,6030,7080$, and $7910 \mathrm{~Hz})$ transmission received on hydrophone 1 with the AUV/source orientation in the broadside $\left(0^{\circ}\right)$ position. At broadside, hydrophone 1 is in the geometrical shadow zone of the forward sphere. Therefore, the initial arrivals at 7910, 7080, and $6030 \mathrm{~Hz}$ correspond to scattering of the direct path within the AUV. These arrivals are followed $6.4 \mathrm{~ms}$ later by stronger multi-path arrivals via surface and tank bottom reflections. This arrival structure can be compared with that recorded on hydrophone 1 when the AUV is rotated $180^{\circ}$, as shown in Fig. 4. The direct path from the source in this case is unimpeded by any part of the AUV. 


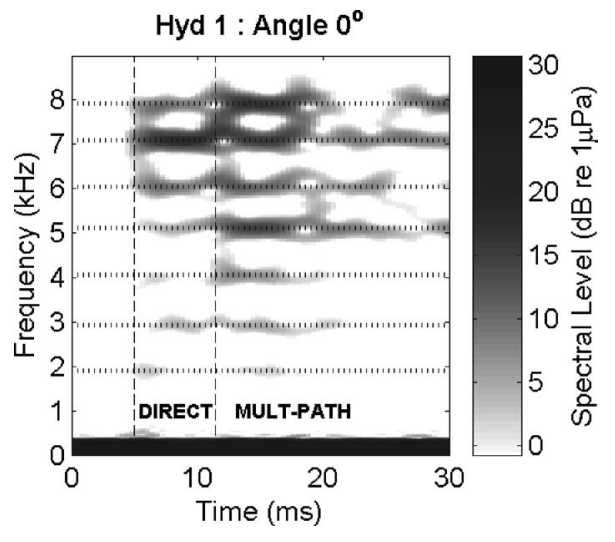

FIG. 3. Spectrogram from hydrophone 1's recording of the mid-frequency comb signal $(1900,2925,4060,5100,6030,7080$, and $7910 \mathrm{~Hz})$ at $0^{\circ}$ incidence angle (broadside to the starboard side).

A pulse duration of $6.4 \mathrm{~ms}$ will propagate an equivilent distance of $9.6 \mathrm{~m}$ in water from beginning to end. This can be compared to the significantly smaller maximum receiver separation in the AUV of $1.55 \mathrm{~m}$. The tank multi-path free pulse duration of $6.4 \mathrm{~ms}$ was therefore considered long enough to allow the interaction of multiple reflections between different parts of the AUV and for a steady-state condition to be reached.

Figure 5 shows the spectral levels of the mid-frequency comb signal $(1900,2925,4060,5100,6030,7080$, and $7910 \mathrm{~Hz}$ ), recorded by hydrophone 1 as a function of incidence angle in azimuth. All the tone levels exhibit an angular interval with high received levels, consistent with direct exposure of the receiver to the source (incidence angles between $110^{\circ}$ and $250^{\circ}$ ). However, the tones also exhibited a degree of variation in received level over this same interval, where the variations have a strong angular, spatial, and frequency dependence. Many of the tones in Fig. 5 show a relatively complex amplitude structure over the complete $360^{\circ}$ sector. In the case of the $7910 \mathrm{~Hz}$ signal, as much as $30 \mathrm{~dB}$ variation in received level can be observed even where the receiver is within the shadow zone of either of the spheres $\left(30^{\circ}-90^{\circ}\right.$ and $\left.280^{\circ}-330^{\circ}\right)$. In general, the dependence of the spectral levels on incidence angle decreases in complexity with decreasing frequency, although all the tones exhibit some angular structure over the entire sector. The

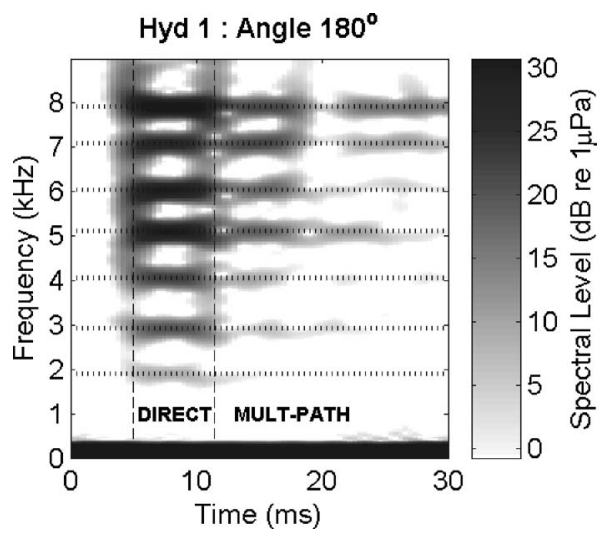

FIG. 4. Spectrogram from hydrophone 1's recording of the mid-frequency comb signal at $180^{\circ}$ incidence angle (broadside to the port side).

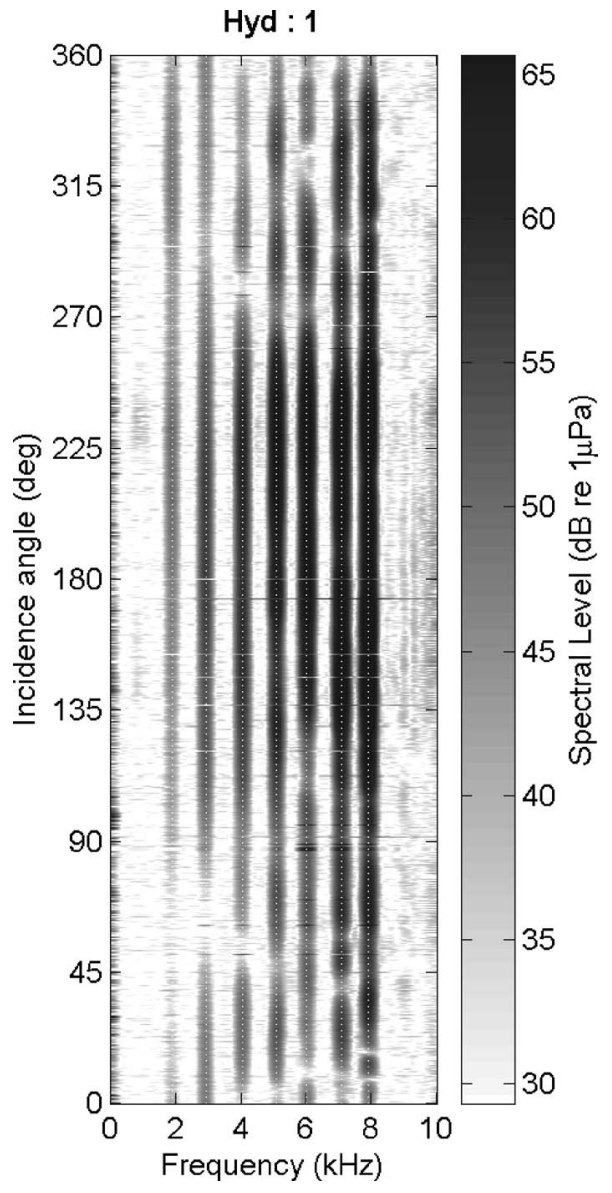

FIG. 5. Mid-frequency comb spectral levels received by hydrophone 1 on the port side of the AUV as a function of angle of incidence over the complete $360^{\circ}$ azimuthal interval.

observed reduction in overall signal level with decreasing frequency is consistent with the frequency dependence of the (ITC-1007) transducer transmit voltage response curve.

For comparison with Fig. 5, Fig. 6 shows the received levels versus frequency and incidence angle for receiver 6 situated approximately on the opposite side of the AUV from hydrophone 1. In this case, the location of the angular interval with high received levels for each of the tones is shifted by nearly $180^{\circ}$ to that of receiver 1 . Again, the incidence angle interval of high received levels for all tones is strongly correlated with the geometric orientation of the source/ receiver direct path and the two glass spheres, suggesting that the spheres are the major contributors to the angular variability of the acoustic field. Figures 5 and 6 clearly illustrate the strong frequency and angular dependence of the spectral levels of the individual tones recorded by a single receiver and the significant differences between the received levels of the same tones on two spatially separated receivers.

\section{NUMERICAL MODELING}

\section{A. Time domain finite difference approach}

A numerical modeling effort was carried out using a time-domain computer code based on a staggered grid, pseudo-spectral finite difference scheme implemented in two spatial dimensions CABRILLO (Gerstoft, 2004). The finite dif- 


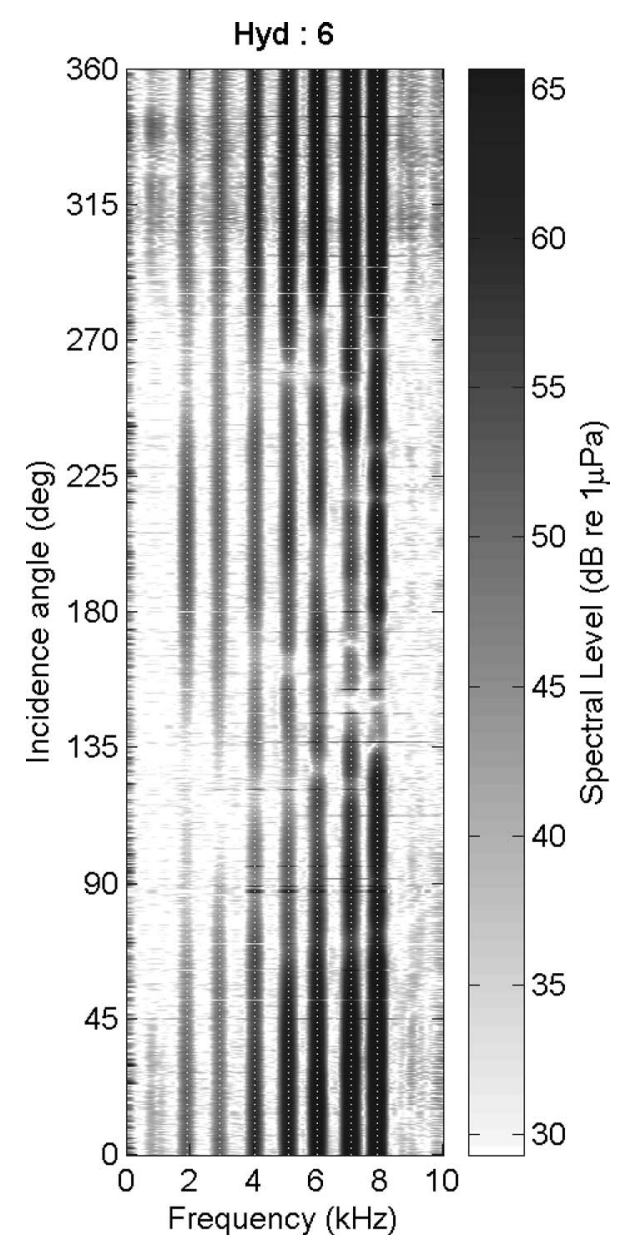

FIG. 6. Mid-frequency comb spectral levels recorded by hydrophone 6 on the AUV's starboard side as a function of angle of incidence in azimuth.

ference method provides the ability to model finite frequency effects in both forward and backward propagation (Fricke, 1993, Tirkas et al., 1993, Stephen, 1996, Wang, 1996, Hastings et al., 1997; and Chen et al., 1998). These full wave field effects are particularly important in describing the complex near-field interference structures observed in the acoustic field near an underwater vehicle.

The CABRILLO code approach to solving the wave equation in the time domain contrasts with most other numerical techniques used in ocean acoustics, such as the Parabolic Equation solution (Collins et al., 1989, 1992, Levy and Zaporozhets, 1998 and Schneider et al., 1998), where the frequency domain Helmholtz wave equation is solved. Calculation of the time-domain solution allows direct comparison with the measured data outlined in Sect. II. CABRILLO is capable of modeling acoustic, elastic, and poro-elastic media. For the purpose of this study, only the surrounding water and the thin shells of the two instrumentation glass spheres were modeled as acoustic media, where the sound speed in glass was set to the value of the glass compressional wave speed. The interior of the glass spheres was modeled as a vacuum with zero sound speed. The 2D modeling approach approximates the two instrumentation glass spheres as fixed cylindrical shells. The use of a staggered grid gives improved numerical accuracy and is better able to handle the large sound speed and density contrasts between the instrumenta-

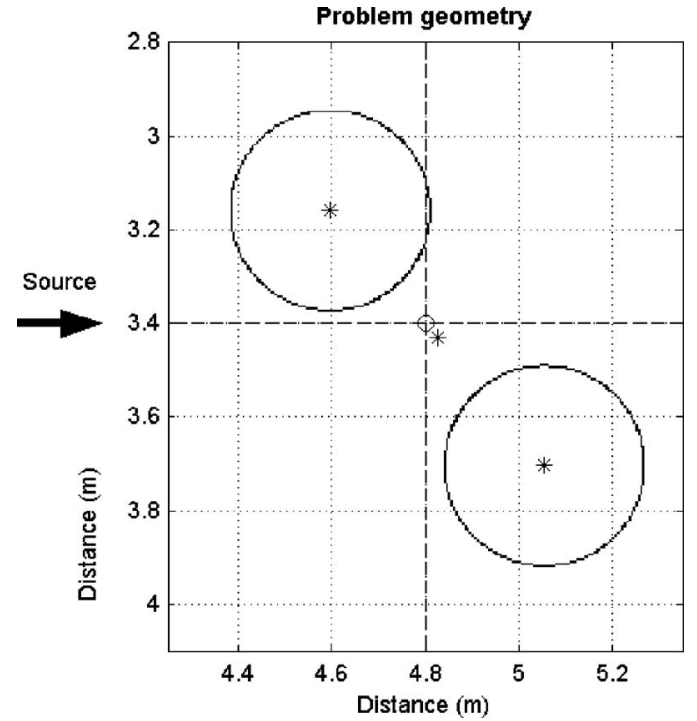

FIG. 7. Geometry for the two-dimensional finite difference modeling of sound scattered from two spherical shells, modeled as cylindrical shells with a vacuum interior, at a $40^{\circ}$ incidence angle. Asterisks mark the centers of each of the two spheres and the midway point along the line between these spheres' centers. The small circle in the center of the plot, offset by $4 \mathrm{~cm}$ from the midway point between the two spheres, indicates the center of rotation of the AUV during the measurements at TRANSDEC. The upper left large circle in the figure represents the aft instrumentation glass sphere in the AUV and the other circle represents the forward AUV glass sphere.

tion spheres of the AUV, the enclosed air, and surrounding water in this problem. In a pseudo-spectral method, the spatial derivatives are solved by multiplication of the wave number in the wave number domain. For a regular staggered grid, the spatial derivative is given by

$$
\frac{\partial U(x)}{\partial x}=F^{-1}\left[i k_{x} e^{\frac{-i k_{x} h}{2}} F\{U(x)\}\right],
$$

where the spatial wave number, $k_{x}$, is

$$
k_{x}=2 \pi j / h \text { for } j=1, \ldots, N \text {. }
$$

The quantity $h$ is the grid spacing, $N$ is the number of grid points, $i$ is $\sqrt{ }-1$, and $F$ represents the Fourier transform. The pseudo-spectral method provides the highest theoretically possible accuracy for a spatial differentiation (Gerstoft, 2004). Euler integration is then used to solve the time derivative using a conventional finite difference approach. For an acoustic medium

$$
\frac{\partial U(x, y, t)}{\partial t}=\frac{1}{2 \Delta t}\left[U^{(n+1)}(i, j)-U^{(n-1)}(i, j)\right],
$$

where $i$ and $j$ are the spatial indices in directions $x$ and $y$, respectively, and $n$ is the temporal index.

\section{B. Problem geometry}

Figure 7 shows the 2D finite difference grid geometry for the two sphere problem. The outer edges of the two shells are placed $0.280 \mathrm{~m}$ apart, corresponding to the separation of the glass instrumentation spheres in the AUV. The TRANSDEC measurement setup was modeled using a single omnidirectional source placed $2.25 \mathrm{~m}$ from the center of AUV rotation, marked by the small circle in the center of Fig. 7 . 

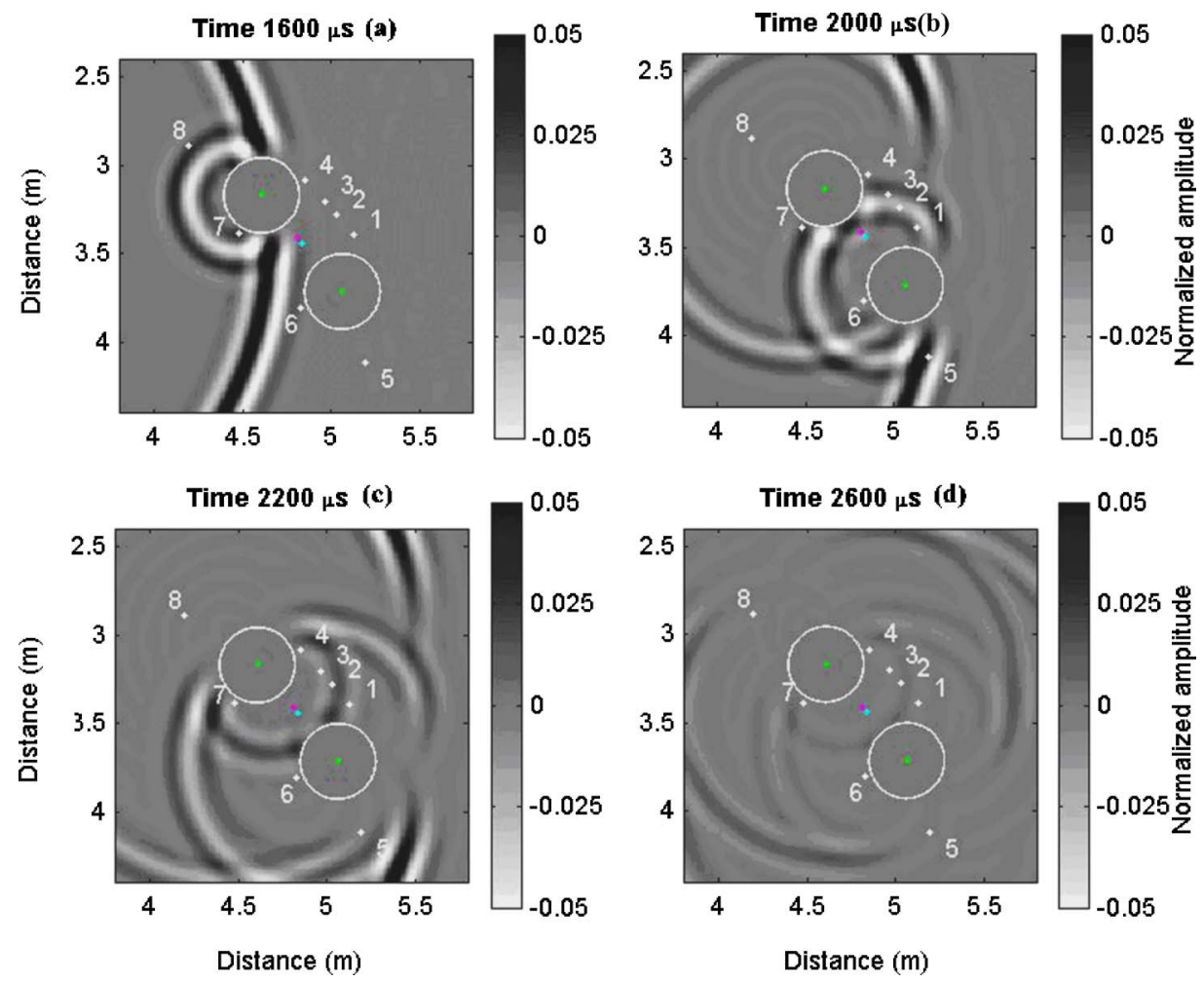

FIG. 8. (Color online) Snapshots of the pressure field amplitude at four different times (listed above each plot) after transmission of a Ricker-type pulse, as calculated by the 2D time-domain finite difference computer code. The point source in each figure is at a range of $2.25 \mathrm{~m}$ to the left from the center of each figure and the bearing from the source to the plots' center (incidence angle) is $40^{\circ}$. The amplitudes in all figures have been normalized by the same value.

(The point equidistant between the centers of the two spheres, marked by an asterisk in Fig. 7, is offset $4 \mathrm{~cm}$ from the center of rotation). The shell density and P-wave velocity were taken as $2300 \mathrm{kgm}^{-3}$ and $5200 \mathrm{~ms}^{-1}$, respectively, representative of the properties of glass. Standard values for water were used for the surrounding fluid. The interior of the shells was assumed to be a void with zero sound speed. For a shell radius of $0.215 \mathrm{~m}$, the product of the wave number and radius $(\mathrm{ka})$ varies from 0.9 to 7.2 over the frequency band $1-8 \mathrm{kHz}$. A time step $d t$ of $0.4 \mu$ s (equivalent to a data sample frequency of $250 \mathrm{kHz}$ ) was used in the modeling and the computations were allowed to advance 15000 steps corresponding to a $6 \mathrm{~ms}$ total propagation time.

\section{Results}

Figures $8(a)-8(d)$ show a series of four snapshots of the acoustic pressure field amplitude (both positive and negative values) as a function of the two spatial dimensions for a spherically spreading $8 \mathrm{kHz}$ Ricker (Ricker, 1953) type source pulse incident at $40^{\circ}$ to the spheres and at a source range of $2.25 \mathrm{~m}$. At $1600 \mu$ s (Fig. 8(a)), the primary wave front interacts with the upper sphere (the aft sphere in the AUV) with a strong reflection back towards the source. As the primary wave front progresses (Fig. 8(b)), diffraction of the primary wave front into the geometrical shadow zone of the upper sphere is evident. In addition, the interaction of the first reflection of the primary wave front from the lower sphere with the first reflection from the upper sphere can be seen around hydrophone 6. Figure 8(c) and 8(d) (the lower two panels) show the development of progressive multiple reflections between the two spheres well after the main wave front has passed. The effects of diffraction also are visible as each reflection passes the opposite sphere. Figure 8(d) shows a fourth-order reflection arrival at the lower sphere just under $1 \mathrm{~ms}$ after the initial wave front passes. However, this multiply reflected signal is $40 \mathrm{~dB}$ below the primary wave front arrival and therefore is considered insignificant in relation to the earlier arrivals.

The individual pressure field solutions at each tone frequency then were calculated from the time domain solutions for a $1 \mathrm{~cm}$ spaced grid over a $2 \mathrm{~m} \times 2 \mathrm{~m}$ area centered on the center of rotation. Figure 9 shows the magnitude of the complex pressure field solution at $7910 \mathrm{~Hz}$ for three incidence angles $\left(0^{\circ}, 50^{\circ}\right.$, and $\left.90^{\circ}\right)$. In the endfire orientation $\left(90^{\circ}\right)$ shown in the lower panel, the interference pattern to the left of both spheres is due to the interaction of the incident signal with the scattered signal from the sphere closest to the source. As the incidence angle is rotated by an increasing amount away from endfire, the effects of scattering from the second sphere become increasingly more significant.

The middle panel $\left(50^{\circ}\right.$ incidence) shows the development of a more complex field structure. In particular, the lower left quadrant of this panel shows scattered energy from the left-hand sphere, the backscattered field from the other sphere, and the direct field mutually interfering with one another (Ingenito, 1987, Carrion et al., 1990; and Stephen, 2000). This asymmetric field becomes symmetrical again as the incidence angle decreases to $0^{\circ}$ (broadside), as observed in the uppermost panel of Fig. 9. 

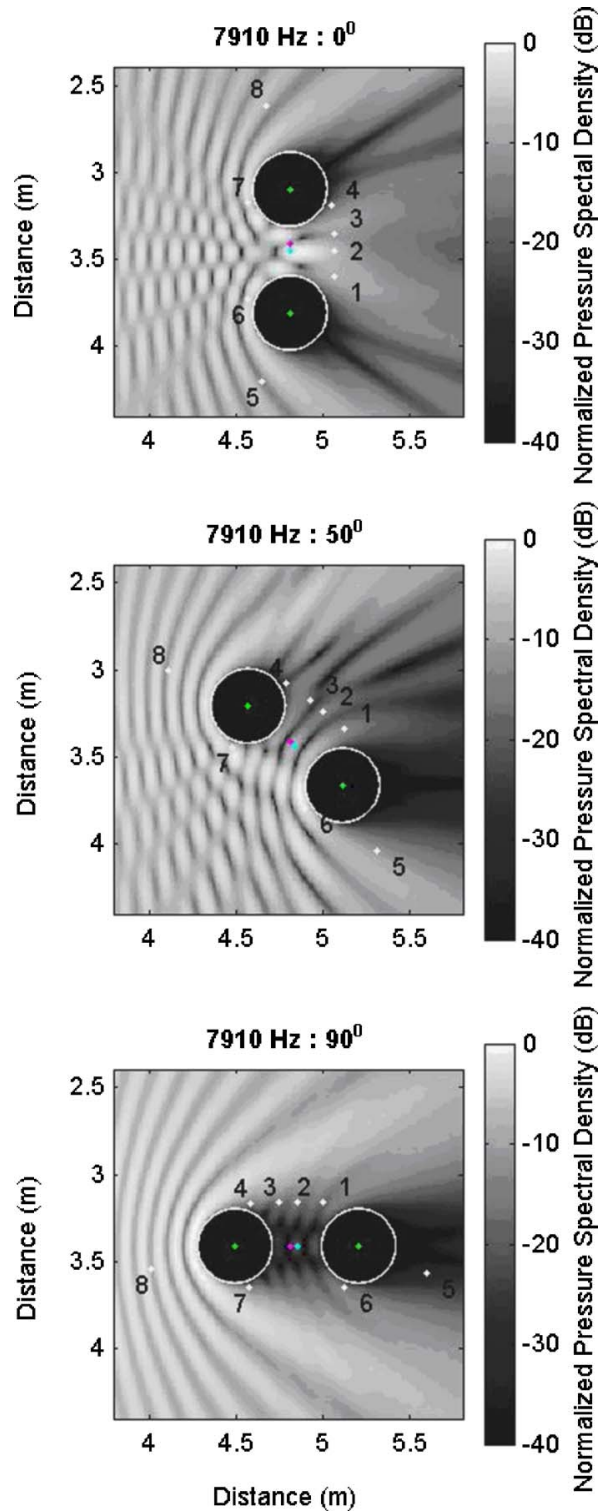

FIG. 9. (Color online) Numerical modeling results for the 2D spatial distribution of the normalized complex pressure field magnitude at $7910 \mathrm{~Hz}$ for an incidence angle of $0^{\circ}$ (upper plot), $50^{\circ}$ (middle plot), and $90^{\circ}$ (lower plot).

Diffraction effects result in energy appearing in the geometrical shadow zone of the two spheres. For $90^{\circ}$ incidence, diffracted energy in the shadow zone of the left-hand sphere then is scattered from the right-hand sphere, resulting in the weak interference field structure in the region of receivers 1-4 and 6,7. At other geometries, more complex field structures may exist in the spheres' shadow zones due to the interaction of both scattered and diffracted energy. As an example, the shadow zone field structure at an incidence of $50^{\circ}$ for the left-hand sphere in Fig. 9 shows considerably more complexity due to scattered energy from the right-hand sphere. A relatively simple shadow zone structure is observed behind the right-hand sphere consistent with standard edge diffraction effects. These results indicate that the multiple-reflected wave fronts, as observed in Fig. 8(d), are weak compared to the diffraction of the primary wave front.

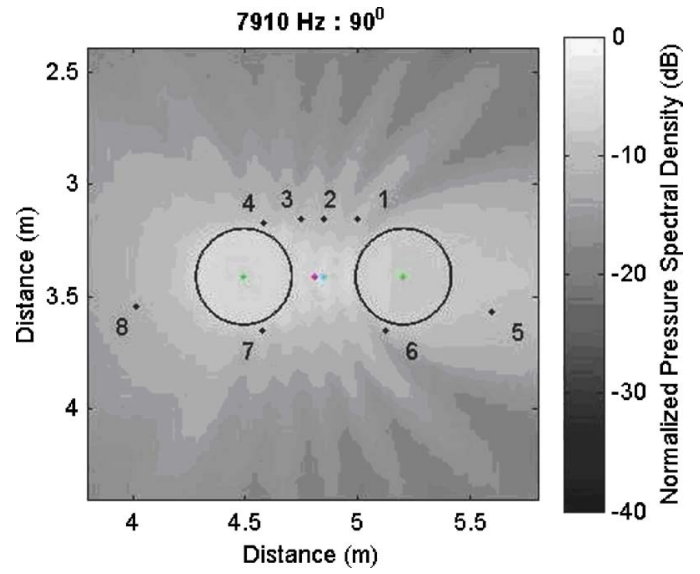

FIG. 10. (Color online) The numerically modeled scattered pressure field magnitude at $7910 \mathrm{~Hz}$ and $90^{\circ}$ angle of incidence. The scattered component is obtained by subtracting the field calculated without any scatterers present from the total field calculated with scatterers present.

The field structure associated only with the interaction of multiple-reflected and diffracted energy can be seen more clearly in Figs. 10 and 11, where the incident pressure field with no scatterers present is subtracted from the total pressure field with scatterers present. In Figs. 10 and 11, the difference field to the left of the spheres is homogeneous since it is composed only of energy scattered from the spheres. The weaker interference patterns due to interaction between diffraction and multiple reflections between the two spheres however is clearly present, particularly in Fig. 11. At $90^{\circ}$ incidence (Fig. 10), the interference structures are quite weak because they arise solely to multiple reflections of energy diffracted from the left-hand sphere. In contrast, a stronger interference field is present in Fig. 11 due the interaction of the primary scattered fields from both spheres.

In summary, Figs. 9-11 illustrate that complex interference field structures can exist in the acoustic field surrounding two glass spheres due to diffraction and multiple scattering. These structures demonstrate a strong spatial and frequency dependence similar to that observed in the measured data from the AUV.

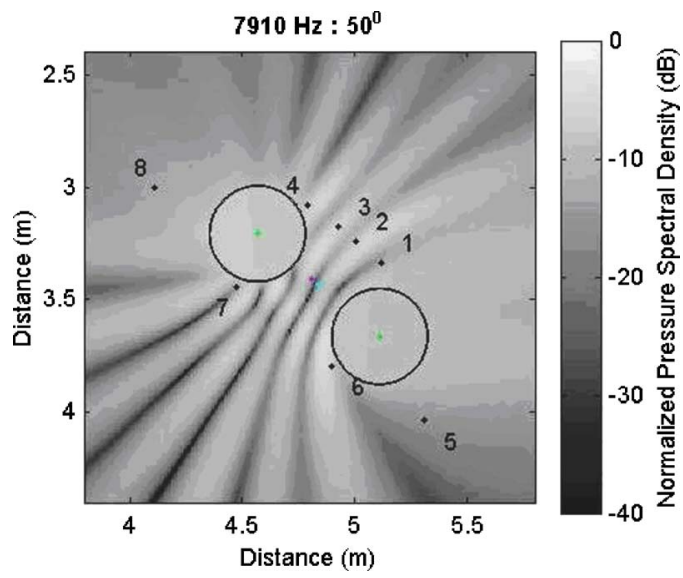

FIG. 11. (Color online) The magnitude of the scattered component of the pressure field at $7910 \mathrm{~Hz}$ and $50^{\circ}$ angle of incidence. 


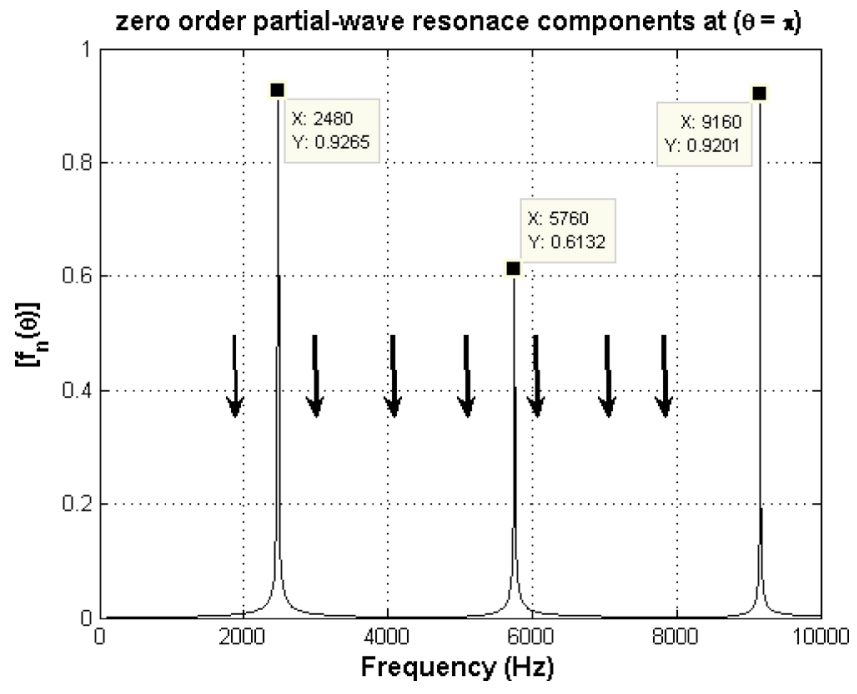

FIG. 12. (Color online) The magnitude of the total field form function (Eq. (4) in the text) for the zeroth mode in the analytical solution for the scattering from an elastic, vacuum-filled, thin-walled spherical shell as a function of frequency. The incident field is a single plane wave, the elastic properties of the shell are those of glass, and the form function is evaluated in the forward-scattered direction $\left(\theta=180^{\circ}\right)$.

\section{COMPARISON OF THE MEASUREMENTS AND NUMERICAL MODELING RESULTS}

In the 2D finite difference calculations presented in the previous section, the three-dimensional AUV instrumentation glass spheres were modeled as two-dimensional infinite cylinders. The cylinders' shells were modeled as an acoustic medium, with a sound speed equal to the compressional speed in glass. In reality, the small value of the ratio of the shell thickness to outer radius, of order $6.5 \%$, suggests the spheres might be effectively modeled as "acoustically soft bubbles," with no intermediate boundary between the outer fluid and the inner void. On the other hand, if the elastic properties of the shell are considered, the resonance characteristics may be altered appreciably from those of a bubble and so could contribute significantly in a different way to the interference fields surrounding the AUV.

To evaluate the potential effects of the elastic properties of the glass shell on the character of scattering, exact solutions for the scattered pressure from a single thin, elastic, spherical shell enclosing a vacuum were implemented as outlined by (Vesker, 1993). The partial-mode form function as a function of angle $\theta$ (the angle with respect to the direction of the incident plane wave signal) is given as

$$
f_{n}(\theta)=\frac{2}{x}(2 n+1) \exp \left(i \delta_{n}\right) \sin \left(\delta_{n}\right) P_{n}(\cos (\theta)),
$$

where $\delta_{n}$ is derived from the fifth-order determinants $D_{n}^{(1)}$ and $D_{n}^{(2)}$ given by Goodman and Stern, 1962, and $P_{n} \cos (\theta)$ is the Legendre polynomial of order $n$. Figure 12 shows the resonance components of the first (zero-order) partial-wave mode for a single AUV instrumentation glass sphere modeled as a 3D thin elastic shell with a vacuum interior. Three resonances exist across the spectrum of interest, at frequencies 2480, 5760, and $9160 \mathrm{~Hz}$. Arrows in Fig. 12 indicate the frequencies of the mid-frequency tones transmitted by the source in this study. These resonances could contribute significantly to the interference field structure around the body of the AUV at source frequencies equal, or close, to the resonance frequencies. Therefore, to achieve a good match between the numerical modeling and data results, the number and frequencies of these resonances must be accurately calculated. The analytical model in Eq. (4) was used to determine the sensitivity of these resonances to changes in the properties of the glass shell. The effect of decreasing the shear wave speed to small values while leaving the compressional speed fixed was to cause a shift in the frequencies of the three resonances downward slightly by a few hundred hertz, suggesting that the elastic properties of the glass play only a minor role. Similarly, setting the compressional and shear wave speeds to that of glass and reducing the thickness of the glass shell to a negligibly small value resulted in a resonance structure identical to that in Fig. 12, but upshifted in frequency by 100-200 Hz. Setting the shear wave speed to that of glass, the glass thickness to its original value, and reducing the compressional wave speed significantly also causes an up-shift in the resonances in the mid-frequency band of interest. In this case, shifts of $1-2 \mathrm{kHz}$ were observed in the mid-frequency band resonance frequencies for a compressional wave speed reduced to around $100 \mathrm{~m} / \mathrm{s}$ above the shear wave speed. In any case, if a realistic value for the compressional speed is used in the modeling, the properties of the glass shell have only a small effect on the scattering resonances. Note that a depth dependence of the resonance frequencies of these spherical shells has been observed in the ocean, believed to be caused by changes in air temperature (and so sound velocity) inside the spherical shell (D'Spain et al., 1991). Calculations showed that an $8 \mathrm{kHz}$ resonance could be shifted by $50 \mathrm{~Hz}$ with an internal temperature variation of $6-2^{\circ} \mathrm{C}$. For this paper, the implementation of a three-dimensional solution for coupled, elastic scattering from two spheres was beyond the scope of the work. However, the effects of elastic scattering may explain some of the observed measured/modeled differences and so should be considered in any future work. Clearly, accounting for the 3D nature of the scattering is important in any future effort. At the least, the results for the two-dimensional case presented in the previous section still are useful in understanding the implications of near-field interference structures in source localization.

Two-dimensional finite difference simulations were run for incidence angles in azimuth over a $360^{\circ}$ sector. Comparison then was made between modeled and measured results at all frequencies. Figure 13 shows the normalized level of the $7910 \mathrm{~Hz}$ signal for hydrophone 1 as a function of incidence angle. The normalized levels from the TRANSDEC measurements are plotted as small diamonds, the finite difference modeling results are shown as connected circles, and the effective mean noise over all channels during the TRANSDEC tests, normalized by the maximum received level over all azimuths, is represented by the horizontal dashed line. An angular interval with high received levels is observed between $100^{\circ}$ and $270^{\circ}$, closely corresponding to the incidence angle interval where hydrophone 1 is exposed directly to the source. Receivers 1-4 on the AUV's port side and 6 and 7 on 


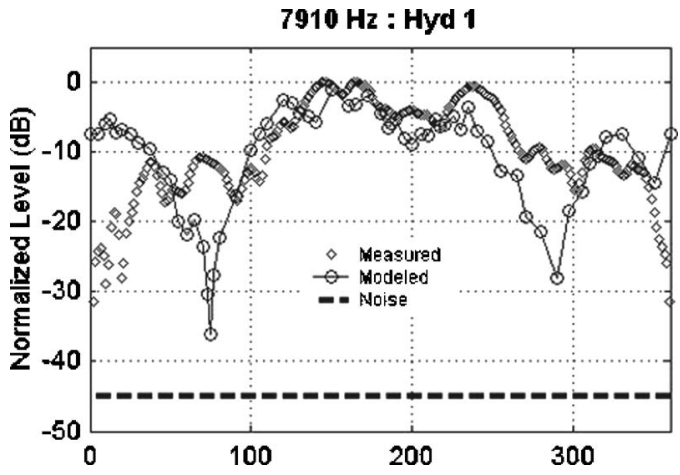

FIG. 13. Comparison of the normalized pressure amplitude at $7910 \mathrm{~Hz}$ for hydrophone 1 as a function of incidence angle from the numerical modeling output (connected circles) and from the TRANSDEC measurements (diamonds), The normalized average electronic self-noise level over all hydrophones in the TRANSDEC measurement effort is plotted as a horizontal dashed line.

the starboard side all exhibit similar correlation between the measured data and the model calculations (re Fig. 14 for hydrophone 7) over the angular interval of high received levels. Variations in hydrophone 1's received levels with changes in incidence angle in the angular interval of high received levels are relatively small, with less than a $6 \mathrm{~dB}$ variation in level from $110^{\circ}$ and $260^{\circ}$. This hydrophone is placed close to the forward sphere in the AUV and so the interference field structure is relatively simple as the incidence angle is varied. A greater degree of complexity with incidence angle, with variations as large as $15 \mathrm{~dB}$, is observed in the modeling results for hydrophone 7 (Fig. 14). This high degree of variability is not present in the measurements. The finite difference model predicts a relatively strong interference pattern between the two spheres (where hydrophone 7 is located) which is a sensitive function of incidence angle. This structure is primarily due to interference of the fields scattered from either sphere and the incident field. The much smoother response observed in the measured data may be due to the much weaker scattering response of a three-dimensional sphere compared to the infinitely long, 2D cylinder used in the modeling (Stanton, 1988 and Stanton et al., 1998). For example, at $k a \gg 1$, a rigid, fixed sphere has an equivalent target strength computed in the back direction (Urick, 1983 and Page et al.,

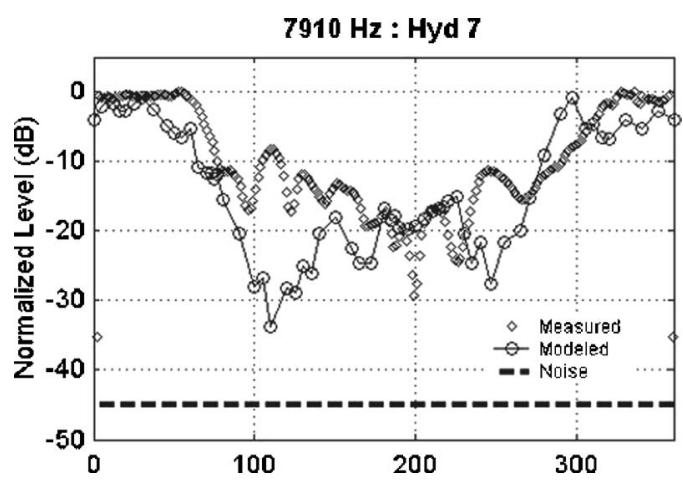

FIG. 14. Comparison of the numerical modeling and measurement results for the normalized amplitude at $7910 \mathrm{~Hz}$ as a function of incidence angle as in Fig. 13, but now for hydrophone 7.
2000) of around $-19 \mathrm{~dB}$ compared with $-6 \mathrm{~dB}$ for a rigid, fixed cylinder. For the weaker target, more of the incident energy will propagate (diffract) around the sphere resulting in less well-defined nulls. At higher frequencies (re Fig. 14), the variability in level over the angular interval of high received levels is less in the measurements than in the model output because the field backscattered from the sphere (measured data) is significantly weaker than that predicted by the modeling (Levy and Zaporozhets, 1998). Additional effects contributing to the measurements such as reflections and scattering from the AUV components other than the two spheres also may effectively smooth over the interference structures predicted by the modeling.

As the incidence angle changes so that the direct source/ receiver path is blocked by one or both of the instrumentation spheres, both modeling results and measurements for most receivers exhibit a more complex field structure; re Figs. 13 and 14. In a few cases, the measurements in this geometrical shadowing regime show a slightly more complex structure than that predicted by the modeling, possibly because of additional interactions from scattered energy from other parts of the AUV. However, the levels in this regime generally are much lower (peaks are 10-20 dB lower) than the levels in the angular interval where the path between the source and receiver is unimpeded by any scatterers. Therefore, at smaller signal-to-noise ratios, much of the finer detail observed in this angular interval would be lost.

Both measurements and modeling results reveal a reduction in the complexity of the field structure with decreasing frequency, consistent with the increase in the acoustic wavelength. The interference structures become less prominent at the lower frequencies and the match between measurement and prediction improves. These results suggest that at lower mid-frequencies, the two glass spheres are the major contributors to the near-field scattering response at all angles of incidence, and that this scattering is reasonably well modeled by the $2 \mathrm{D}$ case. As at higher frequencies, the response with incidence angle at lower frequencies shows strong nulls (sometimes as much as $40 \mathrm{~dB}$ below the main peak) in the interference field structure. Again, the nulls observed in the modeling outputs are deeper than those in the measured data due to the differences in scattering response of a $2 \mathrm{D}$ infinitely long cylinder and a 3D sphere.

\section{SOURCE LOCALIZATION}

Often in source localization problems, multiple equally likely source solutions (ambiguities) exist. However, as more independent information is added to the problem (e.g., greater number of receivers, greater spatial diversity, greater number of frequency components), some or all of the ambiguous solutions can be eliminated, leaving a unique (hopefully correct) source position estimate. Matched field processing techniques (Cox et al., 1987 and D'Spain, 1994) were used in this study to compare a series of replica vectors $\boldsymbol{r}\left(x_{i}\right)$ with measured data $\boldsymbol{d}\left(x_{s}\right)$, where $x_{s}$ represents the desired parameter. In this study, $x$ is the incoming signal incidence angle $\left(\theta_{i, s}\right)$ in azimuth, assumed to equal source bearing. The replica vectors are a subset of all possible solutions 


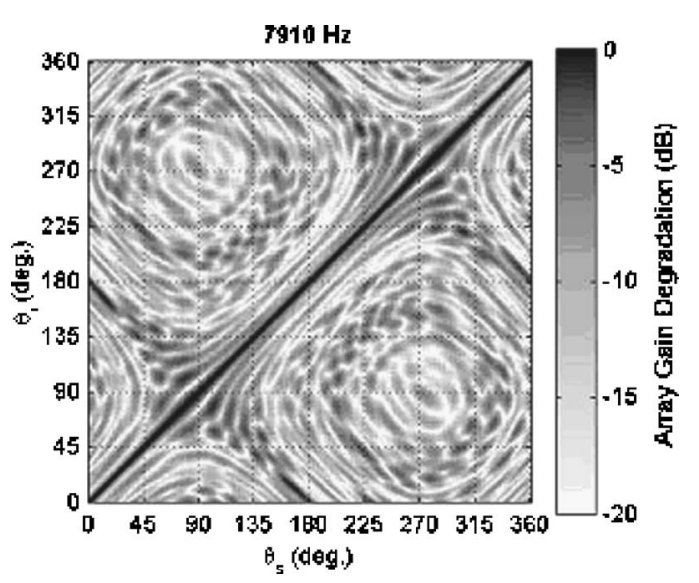

FIG. 15. Array gain degradation (Eq. (5) in the text) for the numerical modeling results at $7910 \mathrm{~Hz}$ compared with themselves in the case where no scatterers are present.

over the full domain of $x$. For narrowband acoustic signals, both amplitude and phase information on multiple receivers can be used to estimate source bearing. In this section, sets composed of complex vectors (the complex acoustic pressure containing both amplitude and phase information), obtained from both measurements at TRANSDEC and numerical model outputs for all eight elements in the AUV hydrophone array, are quantitatively compared with themselves and with each other (they play the role of both replica and data vectors) to allow assessment of array directivity properties and ambiguity resolution for a particular parameter. In addition to complex vector sets formed from the measurements and numerical modeling described in Secs. II and III, an additional vector set was generated using the output from a numerical model in which no scatterers were present (i.e., the two spheres in Sec. III were removed) to evaluate the case of free-field propagation.

A metric based on the conventional Bartlett processor (Kuperman et al., 1990 and Thode et al., 2000), given in Eq. (5), was used to evaluate the comparisons

$$
C\left(\theta_{i}, \theta_{s}\right)=\frac{\left|\boldsymbol{r}\left(\theta_{i}\right) \boldsymbol{d}\left(\theta_{s}\right)^{*}\right|}{\sqrt{\left|\boldsymbol{r}\left(\theta_{i}\right) \boldsymbol{d}\left(\theta_{i}\right)^{*}\right|\left|\boldsymbol{r}\left(\theta_{s}\right) \boldsymbol{d}\left(\theta_{s}\right)^{*}\right|}},
$$

The results then were plotted as ambiguity surfaces on a log scale. The quantity in Eq. (5), which takes on values less than or equal to $0 \mathrm{~dB}$ where $0 \mathrm{~dB}$ signifies an exact match, is referred to as "array gain degradation" and is a quantitative measure of the difference between the "actual" (data) vector and the calculated (replica) vector. Figure 15 shows the array gain degradation for the case of no scatterers present in the model for a signal frequency of $7910 \mathrm{~Hz}$. These modeled results are matched to themselves so that the $0 \mathrm{~dB}$ values along the diagonal from bottom left to top right correspond to matching complex vectors with themselves. However, ambiguities (high side lobes) off this diagonal exist, particularly at incidence angles corresponding to array broadside (near $180^{\circ}$ and $360^{\circ}$ ). Relatively high sidelobe structures are also present around $90^{\circ}$ and $270^{\circ}$, corresponding to endfire source/receiver orientations. In particular, for $\theta_{s}$ equal to $80^{\circ}$, a series of narrow sidelobes on either side of the true value (on the diagonal) covering a $55^{\circ}$ range from $50^{\circ}$ to $105^{\circ}$

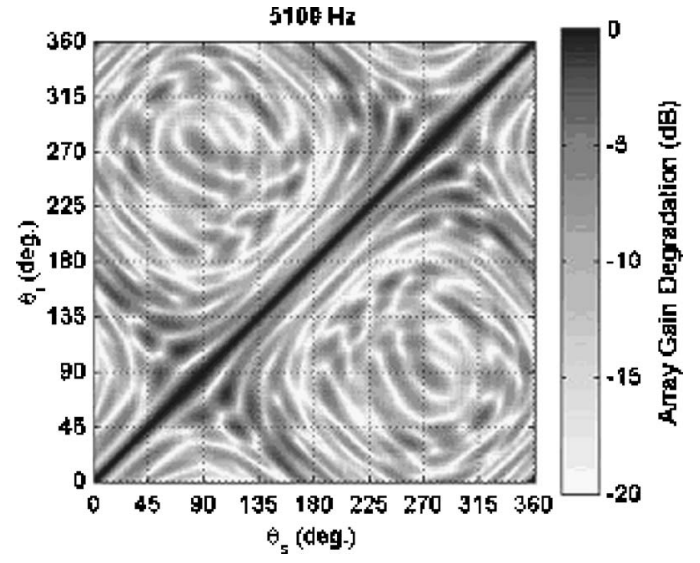

FIG. 16. Array gain degradation for the numerical modeling results compared with themselves in the case with no scatterers present, as in Fig. 15, but now at a frequency of $5100 \mathrm{~Hz}$.

have peaks within $2 \mathrm{~dB}$ of the correct solution. Even worse, the ambiguity ridges at the two broadside positions $\left(180^{\circ}\right.$ and $360^{\circ}$ ) have levels within $0.5 \mathrm{~dB}$ at angle offsets of $\pm 180^{\circ}$ of the true solution (e.g., at a source bearing of $175^{\circ}$, a sidelobe at $7^{\circ}$ is within $0.4 \mathrm{~dB}$ of the true result). Sidelobes with less than $5 \mathrm{~dB}$ array gain degradation are present across the whole range of incidence angles.

The ambiguity surface corresponding to Fig. 15 (numerical modeling with no scatterers present), but at a signal frequency of $5100 \mathrm{~Hz}$, is presented in Fig. 16. The longer wavelength results in a similar but broader sidelobe structure. High-level sidelobes still are present at both the broadside and endfire orientations. The increase in wavelength eliminates any possible phase ambiguities between receivers since the minimum receiver separation $(100 \mathrm{~mm})$ now is smaller than $\lambda / 2$, resulting in a simplified sidelobe structure (but broader main lobe). The array gain degradation plots for all seven individual tone frequencies in the no-scatterer modeling case exhibit a similar sidelobe structure, with the major ambiguities at broadside and endfire, and with a gradual shift to a broader main lobe and fewer sidelobes with decreasing frequency. The sidelobe structure around the endfire orientations has a significant frequency dependence (variation in the number and locations of the sidelobes), whereas the ambiguity structure at broadside is relatively insensitive to changes in frequency.

For comparison with the no-scatterer case, Figs. 17 and 18 present the ambiguity surfaces for the 2D numerical model output at $7910 \mathrm{~Hz}$ with two cylinders present and for the TRANSDEC measurements, respectively. Both of these ambiguity surfaces have similar overall structures, and also have some significant differences with Fig. 15, suggesting that the effects of scattering and shadowing from the two spheres is a major contributor to the directivity properties of the AUV-mounted array. Particularly noteworthy is the near disappearance of the high-level ambiguity ridges present in Fig. 15 at the broadside positions $\left(180^{\circ}\right.$ and $\left.360^{\circ}\right)$. As a specific illustration, in Fig. 17 at $175^{\circ}$ a broad sidelobe exists at $255^{\circ}$ with an approximate level of $-2 \mathrm{~dB}$, and in the measurement case (Fig. 18), broad sidelobes occur at $110^{\circ}$ and $230^{\circ}$ with $-4 \mathrm{~dB}$ levels. In cases where some degree of mis- 


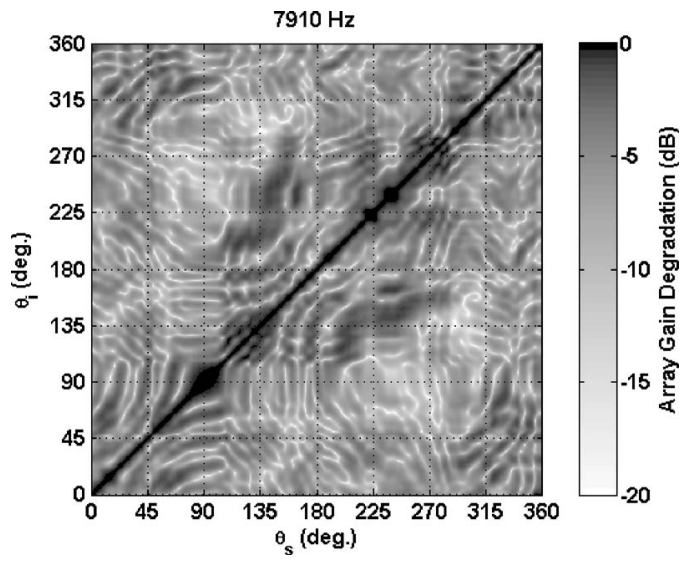

FIG. 17. Array gain degradation for the numerical modeling results at $7910 \mathrm{~Hz}$ compared with themselves in the case where two thin-walled cylindrical shells are present.

match exists, these ambiguities could result in an angular error of $80^{\circ}$ corresponding to the $-2 \mathrm{~dB}$ sidelobe and up to $65^{\circ}$ for the $-4 \mathrm{~dB}$ sidelobe, respectively. In contrast, an error of $180^{\circ}$ can occur in the no-scatterer case for incidence angles near broadside (Fig. 15) because of the corresponding $-0.5 \mathrm{~dB}$ level sidelobes. Similarly, the sidelobe structure around the endfire orientations in the no-scatterer case has a significantly modified appearance in Figs. 17 and 18. In both of these figures, the main-lobe response along the main diagonal broadens. Additional sidelobe structures are present between $0^{\circ}$ and $90^{\circ}$ and $270^{\circ}$ and $360^{\circ}$. These two angular intervals correspond to the orientation of the source/AUV system where the port-side receivers (hydrophones 1-4) are within the geometrical shadow zone of the two glass spheres. This region contains a relatively complex interference field (re Fig. 9) which is primarily due to the interaction of scattered and incident energy and therefore has a strong frequency dependence.

Due to this strong frequency dependence of some of the sidelobe structures, spectral averaging can be used to reduce ambiguities. Figure 19 shows the spectral average for the 7 tones $(7910,7080,6030,5100,4060,2925$, and $1990 \mathrm{~Hz})$ for the no-scatterer case. A general reduction in sidelobe level along with a broadening of the main lobe occurs, re-

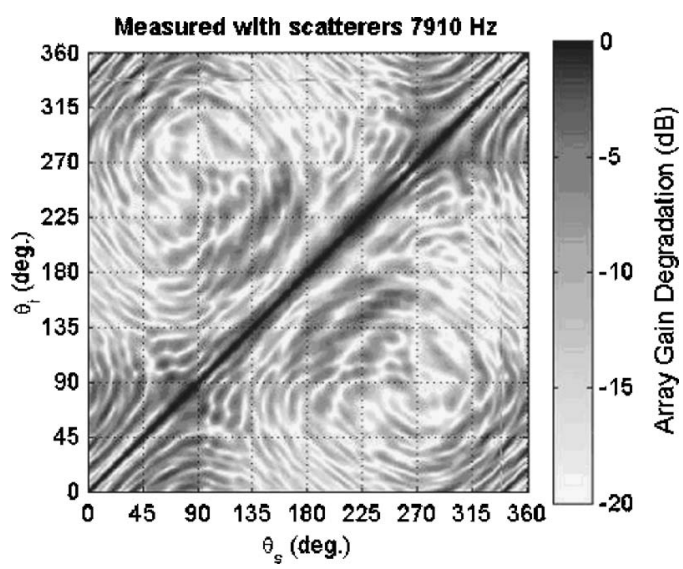

FIG. 18. Array gain degradation for the measurements at TRANSDEC at $7910 \mathrm{~Hz}$ compared with themselves.

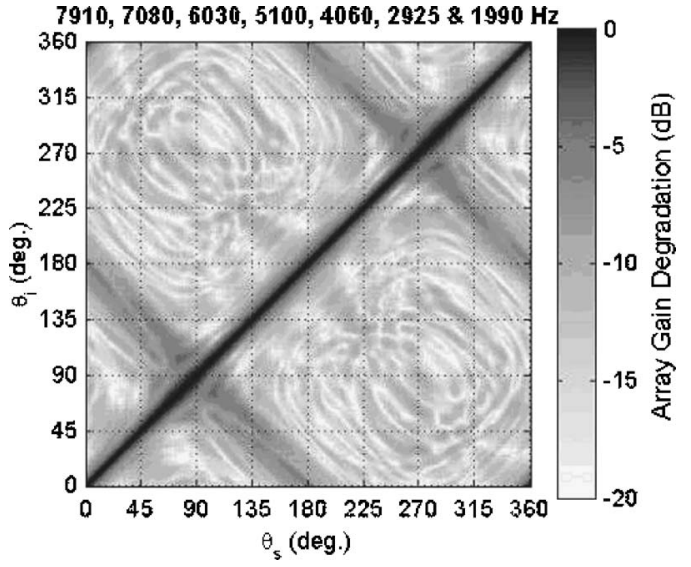

FIG. 19. Array gain degradation averaged over the seven frequencies in the tone comb signal $(7910,7080,6030,5100,4060,2925$, and $1990 \mathrm{~Hz})$ for numerical modeling results compared with themselves in the case of no scatterers present.

sulting in a much clearer definition of the main lobe. For an $82^{\circ}$ incidence angle (near endfire), most of the sidelobe levels are decreased to at least $10 \mathrm{~dB}$ below the level on the main diagonal. A similar reduction in sidelobe level occurs at $175^{\circ}$ (near broadside), although a sidelobe at $-5 \mathrm{~dB}$ level still is present at $7^{\circ}$ which potentially could result in a source bearing estimate error of nearly $180^{\circ}$. This remaining ambiguity appears as the linear gray shading perpendicular to the main diagonal and intersecting it at $90^{\circ}$ and $270^{\circ}$.

Spectral averaging of the array gain degradation across the seven tone frequencies also was carried out for both the numerical modeling with two spheres present (Fig. 20) and for the TRANSDEC measurements (Fig. 21). As with the no-scatterer case, a general reduction in sidelobe level occurs because of the strong spectral dependence of the sidelobe structures. In particular, almost all of the sidelobe structures corresponding to incidence angles where hydrophones $1-4$ are shadowed by one or both spheres $\left(0^{\circ}-90^{\circ}\right.$ and $270^{\circ}-360^{\circ}$ ) and present in the single frequency results (most prominent in Fig. 18) now are reduced to greater than $10 \mathrm{~dB}$ below the main-lobe level. In addition, spectral averaging eliminates the ambiguity at endfire orientations that

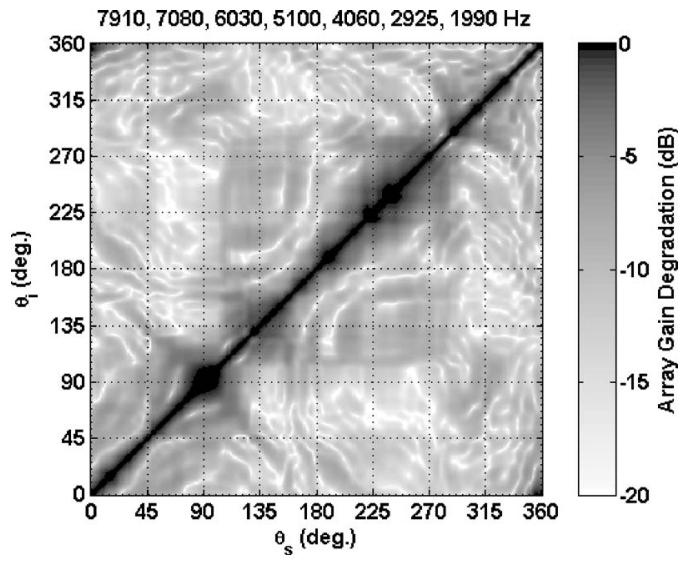

FIG. 20. Array gain degradation averaged over the seven tone frequencies for numerical modeling results compared with themselves in the case of the two scatterers present. 


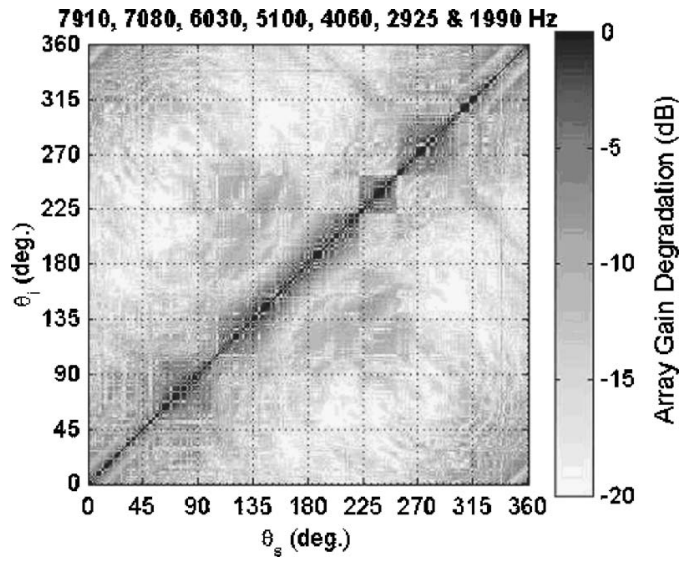

FIG. 21. Array gain degradation averaged over the seven tone frequencies for the measurements at TRANSDEC compared with themselves.

exists in the no-scatterer case. In the spectral-averaged noscatterer case, the maximum sidelobe level is $5 \mathrm{~dB}$ below the main-lobe response, which is reduced to around $8 \mathrm{~dB}$ below in the modeling-with-scatterers case and $10 \mathrm{~dB}$ below in the TRANSDEC measurements. The greater reduction in sidelobe levels for the measurements compared to those for the numerical model output with two spherical shells present is the result of additional frequency-dependent scattering effects by other vehicle components and/or elastic resonance effects of the two glass instrumentation spheres. In general, however, the highest-level sidelobes away from the main diagonal in both Figs. 20 and 21 are approximately $4-5 \mathrm{~dB}$ lower than those in the no-scatterer case. Therefore, the presence of the scatterers leads to enhanced array performance for source localization in azimuth.

The focus of this paper is on the effects of scattering from an object such as an AUV on source localization performance of a hydrophone array in the near field of the scattering object. However, to take full advantage of the increase in complexity of the received field due to this scattering, the numerical model must accurately predict this complexity. Figures 13 and 14 and the discussion in Sec. IV suggest that some of the major scattering features observed in the AUV measurements at TRANSDEC can be modeled using a simple two-dimensional numerical model of two spherical shells (actually cylindrical shells). Quantitative comparison of this model and measured data can be made using the array gain degradation in Eq. (5). Figure 22 shows the array gain degradation for the TRANSDEC measurements versus the 2D model results for the $7910 \mathrm{~Hz}$ tone. Mismatch now exists as measured by array gain degradation values less than $0 \mathrm{~dB}$ along the main diagonal. However, Fig. 22 still demonstrates a relatively strong main-lobe response even with this simplified 2D model. In comparison with the no-scatterer case (Fig. 15), the high-level sidelobe structures around $90^{\circ}$ and $270^{\circ}$ incidence angles and the high-level ambiguity ridges at the broadside positions $\left(0^{\circ}\right.$ and $\left.180^{\circ}\right)$ are significantly reduced. The main lobe in Fig. 22 is broader than in either Fig. 17 or Fig. 18, but its levels still are everywhere higher than those of the associated sidelobes. Some fine sidelobe structure is present between $270^{\circ}-360^{\circ}$ and $0^{\circ}-90^{\circ}$ corresponding with the structures in the measurement-only array gain degrada-

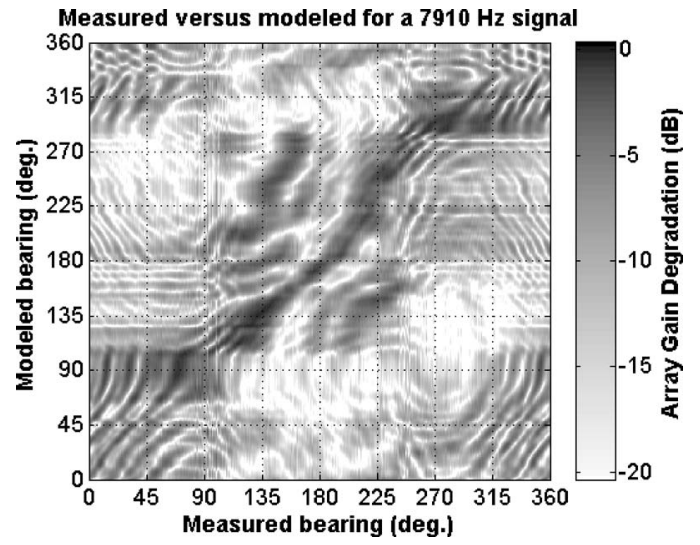

FIG. 22. Array gain degradation at $7910 \mathrm{~Hz}$ for the numerical modeling results with scatterers present compared to the TRANSDEC measurements.

tion plot (Fig. 18). The variability of these structures, however, decreases with increasing wavelength as illustrated in Fig. 23 for a measurement versus model comparison at $6030 \mathrm{~Hz}$. The main lobe in Fig. 23 still occurs approximately in the correct location along the main diagonal, indicating a significant degree of correct processor performance. Therefore, enhancement in certain aspects of localization performance still is achieved in this simplified modeling case over that for the no-scatterer case (Figs. 15 and 16) due to suppression of the high-level sidelobe structures.

\section{CONCLUSIONS}

The matched field processing results in the previous section, as quantified by array gain degradation, show that scattering from the AUV, if not taken into account, adversely affects the localization performance of the AUV hullmounted hydrophone array. On the other hand, knowledge of the scattering patterns around the AUV is useful in reducing ambiguities that would exist if the scatterers were not present. Both results from a $2 \mathrm{D}$ time domain, finite difference numerical model and measurements from an AUVmounted eight-element hydrophone array collected in a large calibration tank show complex field structures due to scattering from the AUV. These scattering features are strongly dependent on incidence angle and therefore contain informa-

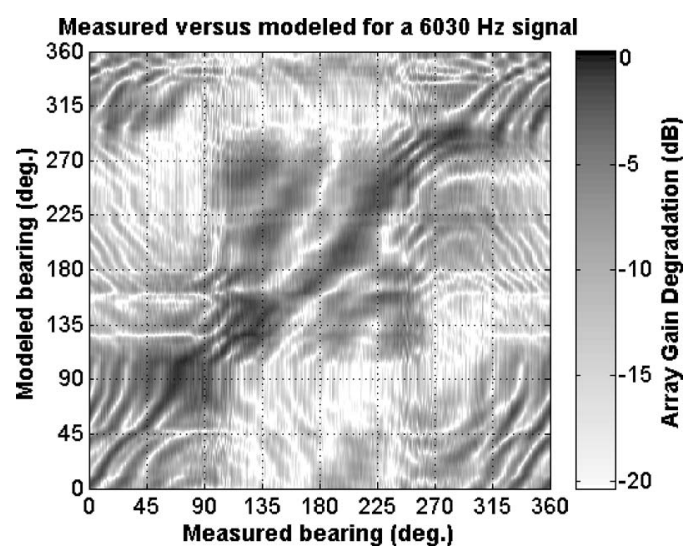

FIG. 23. Array gain degradation at $6030 \mathrm{~Hz}$ for the numerical modeling results with scatterers present compared to the TRANSDEC measurements. 
tion on the direction to the source. The array gain degradation plots demonstrate that if the scattering features are taken into account, the overall level of the sidelobes decreases and the sidelobes tend to reorient themselves closer to the main lobe, thereby enhancing source localization capabilities. In addition, with the introduction of scatterers, most of the sidelobe structures in the array gain degradation plots become strongly dependent upon frequency so that averaging across frequency further reduces sidelobe levels.

Shadowing effects due to the two instrumentation glass spheres in the AUV appear dominant in generating the structure of the received amplitude as a function of incidence angle. An angular interval with high received levels is observed in the data from all hydrophones and at all frequencies. This angular interval is highly dependent upon the receiver position in relation to the source-scatterer configuration-it occurs when the receiver is located between the source and the two glass spheres-and is relatively frequency independent. Even in low signal-to-noise ratio conditions, this structure is useful in reducing ambiguities in source localization that would exist if the scatterers were not present. Additional received field complexity is evident in the TRANSDEC measurements and is most likely due to additional scattering interactions from other parts of the AUV assembly and elastic scattering effects from the two spheres. This additional complexity shows a strong frequency dependence and therefore any associated sidelobe structure in the array gain degradation plot can be reduced significantly by spectral averaging. In low signal-to-noise ratio situations, much of the information in the field structures at lower level may be lost. However, good resolution on the correct source bearing at all angles of incidence without high-level ambiguities using the received field structure over the angular interval where the receiver was positioned between the source and AUV body still can be obtained at lower signal-to-noise ratio for the band of mid-frequency tones used in this study. A reduction of $5 \mathrm{~dB}$ in maximum sidelobe level was observed in comparison with an identical array configuration in an acoustic field without scatterers present.

The numerical modeling results provide valuable insight into the scattering and diffraction effects around the AUV body. One example illustrated by the modeling results is that multiple reflections between the two scatterers are significantly weaker than the diffraction of the incident wave front. Comparison between the numerical modeling results and the measurements shows that a good correlation exists in the locations of the angular intervals with the highest received levels for all hydrophones. This result suggests that much of this structure is dictated by the scattering effects of the two glass spheres. The agreement between the numerical model predictions and the measurements steadily improves with decreasing frequency, probably because the corresponding increase in acoustic wavelength reduces the scattering contributions from AUV components not included in the model. Variations in the levels in the angular interval with high received levels that are present in the modeling results but not in the measured data are most likely due to difference in backscattering strength of the scatterers in the two cases.
That is, in the numerical model, each of the two glass spherical shells is modeled as a 2D cylinder with a thin fluid shell and a vacuum interior. As illustration of the impact of modeling a 3D object with a 2D approximation, a rigid, 2D cylinder has a target strength in the backscattered direction $13 \mathrm{~dB}$ greater than a rigid, 3D sphere $(-6 \mathrm{~dB}$ vs $-19 \mathrm{~dB}$, respectively) for wave number/radius products much greater than unity $(k a \gg 1)$. For $k a$ values around unity, the elastic properties of a spherical glass shell can create scattering resonances that greatly increase the scattering cross section over that of an identically sized rigid sphere. The frequencies of the tones transmitted by the source during the tank measurements do not correspond to the three resonance frequencies of the lowest radial mode of a single vacuum-filled glass shell as calculated by an analytical model; however, they both fall in the same frequency $(1-10 \mathrm{kHz})$ band. Therefore, the resonances must be modeled correctly in order to accurately predict the scattered field. Scattering from additional AUV components that contribute to the measurements but not included in the model also could be a source of discrepancy between the numerical model ouputs and the tank measurements. Future numerical modeling efforts most likely would show significantly improved agreement with the measurements by taking these effects into account. In any case, the modeling results in this paper demonstrate the potential improvement in source localization performance due to increased complexity of the received acoustic field caused by scattering from an object such as an AUV near the receiving array.

\section{ACKNOWLEDGMENTS}

Richard Zimmerman and the Deep Tow engineering group at the Marine Physical Laboratory installed the eightelement hydrophone array and digital data acquisition system in the AUV and conducted the measurement effort at TRANSDEC. Dave Ensberg, Marine Physical Lab, and Howard McManus at the Space and Naval Warfare Center, San Diego, also helped make the TRANSDEC measurements. Peter Gerstoft, also at the Marine Physical Lab, provided us with the Cabrillo time domain finite difference code and thorough documentation on how to use it. Thanks to Bryan Woodward, Loughborough University (UK), for assistance in manuscript preparation. This work was supported by the Office of Naval Research, Codes 321(US) and 321(OA).

\section{APPENDIX: EFFECTS OF VARIATION IN ARRAY POSITION}

To illustrate the impact of variation in array position, the set of complex pressure vectors obtained from the numerical modeling with the hydrophone array in its original position was matched with the complex pressure vector set obtained after the array position was offset by various amounts and in various directions in the horizontal plane, i.e., in the plane of Fig. 9. (Note that since the shape of the array remains unchanged during an offset, the effect on the phase differences between the elements of the array is minimized, thereby decreasing the role of phase in this sensitivity analysis). Figure 24 shows the array gain degradation for a $6030 \mathrm{~Hz}$ signal 


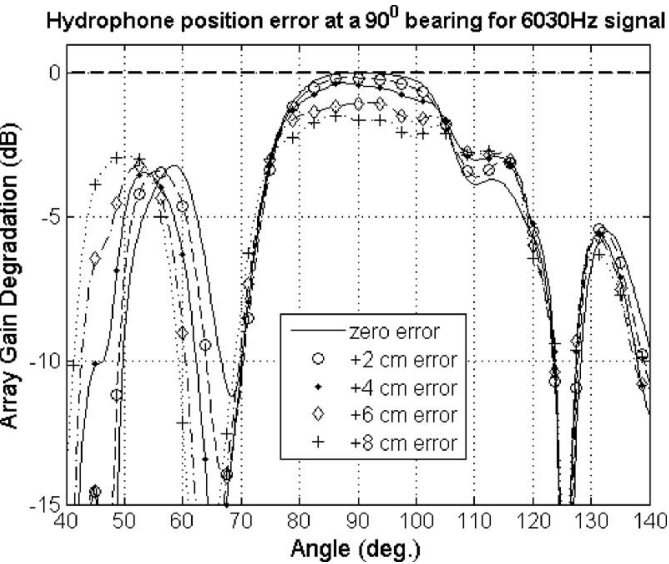

FIG. 24. Array gain degradation as a function of assumed angle of incidence given the true incidence angle is $90^{\circ}$ for hydrophone position errors of $0-8 \mathrm{~cm}$ in $2 \mathrm{~cm}$ increments. The source frequency is $6030 \mathrm{~Hz}$.

arriving at a bearing of $90^{\circ}$ (endfire as shown in the lowermost panel in Fig. 9) for array offsets of $0-8 \mathrm{~cm}$ in $2 \mathrm{~cm}$ increments. All offsets in Fig. 24 were in the direction parallel to the line connecting the centers of the two spheres. The zero-offset plot shows the broad mainlobe structure observed at $90^{\circ}$ (re Fig. 17 for $7910 \mathrm{~Hz}$ ) with no significant sidelobes within $3 \mathrm{~dB}$ of the main lobe. For array offsets up to $8 \mathrm{~cm}$, little degradation in the mainlobe structure occurs; the maximum decrease in mainlobe level at $8 \mathrm{~cm}$ offset is only $2 \mathrm{~dB}$. A corresponding decrease in the mainlobe to sidelobe ratio occurs, from $3 \mathrm{~dB}$ at zero offset to about $1 \mathrm{~dB}$ at $8 \mathrm{~cm}$. This significant tolerance to array offset is the result of the fact that very little change in the acoustic field structure occurs in the direction parallel to the line connecting the centers of the two spheres for an incidence angle at endfire, as seen in the lower panel in Fig. 9. For comparison, Fig. 25 shows the array gain degradation for a source incidence angle of $180^{\circ}$ (broadside). In this case, significant changes in the acoustic field structure occur for offsets in array location along the direction parallel to the line connecting the two spheres, as illustrated in the upper panel of Fig. 9 (which is for an incidence angle of $0^{\circ}$ ). The mainlobe to sidelobe ratio in Fig. 25 decreases from around $+4 \mathrm{~dB}$ for zero offset to



FIG. 25. Array gain degradation as a function of assumed angle of incidence for various element position offsets as in Fig. 24, but now for a true incidence angle of $180^{\circ}$. around $+1 \mathrm{~dB}$ at $4 \mathrm{~cm}$ offset. At $6 \mathrm{~cm}$, the degradation in the main lobe of nearly $10 \mathrm{~dB}$ results in mainlobe levels around $4 \mathrm{~dB}$ below the highest sidelobes; at $8 \mathrm{~cm}$ offset, a deep null appears at the original location of the main lobe. The acoustic wavelength at $6030 \mathrm{~Hz}$ is around $24.8 \mathrm{~cm}$, so that an $8 \mathrm{~cm}$ offset corresponds to a third of a wavelength, sufficient to cause significant changes in the field structure recorded by the elements of the array.

These results illustrate that the sensitivity of the matched field processing output to array position offsets is highly dependent upon the variability of the field structure in the direction of the offset.

Blauert, J. P. (1983). Spatial Hearing, MIT Press, Cambridge, MA.

Butler, R. A., and Balendiuk, K. (1997). "Spectral cues utilized in the localization of sound in the median sagittal plane," J. Acoust. Soc. Am. 61(5), 1264-1269.

Carrion, P. M., and de Brito, M. A. P. (1990). "Resonance scattering in waveguides: Acoustic scatterers," J. Acoust. Soc. Am. 87, 1062-1069.

Chen, Y., Chew, W. C., and Liu, Q. (1998). "A three-dimensional finite difference code for the modeling of sonic logging tools," J. Acoust. Soc. Am. 103, 702-712.

Collins, M. D., and Werby, M. F. (1989). "A parabolic equation model for scattering in the ocean," J. Acoust. Soc. Am. 85, 1895-1902.

Collins, M. D., and Evans, R. B. (1992). "A two-way parabolic equation for acoustic backscattering in the ocean," J. Acoust. Soc. Am. 91, 1357-1368.

Cox, H., Zeskind, R. M., and Owen, M. M. (1987). "Robust adaptive beamforming," IEEE Trans. Acoust., Speech, Signal Process. 10, 1365-1376.

D'Spain, G. L., Hodgkiss, G. L., and Edmonds, G. L. (1991). "The simultaneous measurement of imfrasonic acoustic partical velocity and acoustic pressure in the ocean by freely drifting swallow floats," IEEE J. Ocean. Eng. 16(2), 195-207.

D'Spain, G. L. (1994). "Relationship of underwater acoustic intensity measurements to beamforming," Can. Acoust. 22, 157-158.

Fricke, J. R. (1993). "Acoustic scattering from elemental Artic ice features: Numerical modeling results," J. Acoust. Soc. Am. 93, 1784-1796.

Gerstoft, P. (2004). "CABBRILLO 1.0: Acoustic, elastic and poroelastic finite difference modeling," (http://www.mpl.ucsd.edu/people/gerstoft/ cabrillo/cabrillo.html), last viewed 17th May (2007).

Goodman, R. R., and Stern, R. (1962). "Reflection and transmission of sound by elastic spherical shells," J. Acoust. Soc. Am. 34, 338-244.

Hastings, F. D., Schneider, J. B., and Broschat, S. L. (1997). "A finitedifference time-domain solution to scattering from a rough pressurerelease surface," J. Acoust. Soc. Am. 102, 3394-3400.

Ingenito, F. (1987). "Scattering from an object in a stratified medium," J. Acoust. Soc. Am. 82, 2051-2059.

Kuperman, W. A., Collins, M. D., Perkins, J. S., and Davis, N. R. (1990). "Optimal time-domain beamforming with simulated annealing including application of a priori information," J. Acoust. Soc. Am. 88, 1802-1810.

Levy, M. F., and Zaporozhets, A. A. (1998). "Target scattering calculations with the parabolic equation method," J. Acoust. Soc. Am. 103, 735-741.

Luebbers, R. J., and Beggs, J. (1992). "FDTD analysis and experimental measurements," IEEE Trans. Antennas Propag. AP-40, 1403-1407.

Morse, P. M., and Ingrad, K. U., (1986). Theoretical Acoustics, Princeton University Press, Princeton, NJ, ISBN 0-691-08425-4.

Page, S. J., Brothers, R. J., Murphy, K. M., Elston, G. R., and Bell, J. M. (2000). "A hybrid ray-trace/finite difference model for target strength evaluation," in Proceedings Fifth European Conference on Underwater Acoustics, edited by M. E. Zakharia, P. Chevret, and P. Dubail, Lyon, France, 15-20.

Ricker, N. (1953). "The form and laws of propagation of seismic-wavelets," Geophysics, 18, 10-40.

Schneider, J. B., Wagner, C. L., and Kruhlak, R. J. (1998). "Simple conformal methods for finite-difference time-domain modeling of pressurerelease surfaces," J. Acoust. Soc. Am. 104, 3219-3225.

Skelton, E. A., and James, J. H. (1997). Theoretical Acoustics of Underwater Structures, Imperial College Press, ISBN 1-86094-085-4.

Stanton, T. K. (1988). "Sound scattering by cylinders of finite length fluid cylinders," J. Acoust. Soc. Am. 83, 55-63.

Stanton, T. K., Wiebe, P. H., and Chu, D. (1998). "Differences between sound scattering by weakly scattering spheres and finite-length cylinders 
with applications to sound scattering by zooplankton," J. Acoust. Soc. Am. 103, 254-264.

Stephen, R. A. (1996). "Modeling sea surface scattering by the time-domain finite-difference method," J. Acoust. Soc. Am. 100, 2070-2078.

Stephen, R. A. (2000). "Optimum and standard beam widths for numerical modeling of interface scattering problems," J. Acoust. Soc. Am. 107, 1095-1102.

Tirkas, P. A., Balanis, C. A., Purchine, M. P., and Barber, G. C. (1993) "Finite difference time domain method for electromagmetic radiation, interference, and interactions with complex structures," IEEE Trans. Electromagn. Compat. EMC- 35, 192-203.

Thode, A. M., Kuperman, W. A., D’Spain, G. L., and Hodgkiss, W. S. (2000). "Localization using Bartlett matched-field processor sidelobes," J.
Acoust. Soc. Am. 107(1), 278-286.

Urick, R. J. (1983). Principles of Underwater Sound (McGraw-Hill, New York, 1983).

Vesker, N. D. (1993). Resonace Acoustic Spectroscopy (Springer-Verlag, Berlin).

Wang, S. (1996). "Finite-difference time-domain approach to underwater acoustic scattering problems," J. Acoust. Soc. Am. 99, 1924-1931.

Yee, K. S. (1966). "Numerical solution of initial boundary value problems involving Maxwell's equation in isotropic media," IEEE Trans. Antennas Propag. AP-14, 302-307.

Zimmerman, R., D'Spain, G. L., and Chadwell, C. D. (2005). "Decreasing the radiated acoustic and vibration noise of a mid-size AUV," IEEE J. Ocean. Eng. 30(1), 179-187. 